\title{
Modelo de gestión de inventarios para empresas comerciales de productos agrícolas
}

Inventory management model for commercial enterprises of agricultural products

\author{
Katherine Janeth Tamayo Guzmán \\ ktamayog@psg.ucacue.edu.ec \\ Universidad Católica de Cuenca, Cuenca \\ Ecuador \\ https://orcid.org/0000-0002-8027-3871 \\ Cecilia Ivonne Narváez Zurita \\ inarvaez@ucacue.edu.ec \\ Universidad Católica de Cuenca, Cuenca \\ Ecuador \\ https://orcid.org/0000-0002-7437-9880 \\ Juan Carlos Erazo Álvarez \\ jcerazo@ucacue.edu.ec \\ Universidad Católica de Cuenca, Cuenca \\ Ecuador \\ https://orcid.org/0000-0001-6480-2270
}

Recibido: 07 de septiembre de 2019

Aprobado: 28 de septiembre de 2019

\begin{abstract}
RESUMEN
Para las empresas comerciales los inventarios representan inversiones de mayor importancia en relación con los demás activos, ya que son fundamentales para las ventas y para la obtención de utilidades, la unidad de análisis de la presente investigación es la empresa comercial "Agroinsumos SV" dedicada a la comercialización de productos agrícolas situada en la ciudad de Machala provincia de El Oro, en la cual se evidencian problemas en el control de sus inventarios. Razón por la cual se propone un Modelo de gestión de inventarios que incorpore elementos de la administración efectiva de inventarios. La metodología se desarrolló bajo el enfoque mixto, por cuanto
\end{abstract}


en su ejecución se obtuvo información cuali - cuantitativa. Fue transversal con un alcance descriptivo - explicativo. Los resultados más relevantes tienen relación con la necesidad imperiosa de establecer mecanismos que permitan determinar las existencias y realizar un análisis de inventarios.

Descriptores: Modelo; Inventarios; Gestión; Eficiencia; Análisis.

\begin{abstract}
For commercial companies, inventories represent investments of greater importance in relation to other assets, since they are fundamental for sales and for obtaining profits, the unit of analysis of this research is the commercial company "Agroinsumos SV" dedicated to the commercialization of agricultural products located in the city of Machala province of El Oro, in which problems are evidenced in the control of their inventories. Reason why an Inventory Management Model is proposed that incorporates elements of effective inventory management. The methodology was developed under the mixed approach, since in its execution qualitative and quantitative information was obtained. It was transversal with a descriptive - explanatory scope. The most relevant results are related to the imperative need to establish mechanisms to determine stocks and perform an inventory analysis.
\end{abstract}

Descriptors: Model; Inventories; Management; Efficiency; Analysis.

\title{
INTRODUCCIÓN
}

Ecuador es un país altamente comercial y agricultor, en donde el $39 \%$ de las compañías se dedican al comercio y el $14,7 \%$ a la agricultura y ganadería, siendo el banano el primer producto que lo caracteriza, seguido del camarón y finalmente el cacao, conforme cifras del Instituto Nacional de Estadística y Censos existen 454 empresas por cada 10.000 habitantes y de entre ellas, el $89 \%$ son microempresas generando miles de plazas laborales (Expreso, 2014). En tal sentido, el número de empresas dedicadas a la comercialización de productos agrícolas se han incrementado a gran medida en los últimos tiempos, compitiendo ferozmente por su posicionamiento en el mercado.

La dependencia de la importación neta de fertilizantes ha consolidado oligopolios en empresas encargadas de la importación y la comercialización de estos insumos como Agripac, Fertisa y Fertilizantes del Pacífico (Ferpacific). Según cifras del MAGAP, entre 
el periodo 2007-2011, la empresa Fertisa fue la principal abastecedora del mercado nacional de fertilizantes, con una participación en el mercado de $30 \%$, seguida por el Banco Nacional de Fomento (BNF) con 22\% de participación; Ferpacific Noboa con 16\%; Delcorp con 14\%; Brenntag con una participación de 11\%; Agripac con 4\%, y por último, Pronaca con 3\% de participación en el mercado nacional. (MAGAP, citado en, Aillón y Milques, 2012).

La unidad de análisis de la presente investigación es la empresa "Agroinsumos SV", dedicada a la comercialización de productos agrícolas, está situada en la ciudad de Machala provincia de El Oro. Respecto al manejo del inventario es fundamental para alcanzar un equilibrio de existencias, evitar quiebres de stock, garantizar un retorno de la inversión y conseguir altos niveles de servicio, sin embargo, a través de los años estos inventarios han crecido, mas no de una forma organizada lo cual ha ocasionado que actualmente exista una acumulación de productos con alto nivel de inventario, otros considerados caducados y también insumos con baja rotación. Esto genera mensualmente costos varios por almacenamiento, mano de obra y bodegaje.

Por ello, el presente trabajo de investigación tiene por objetivo elaborar un modelo de gestión de inventarios que permita a la empresa mejorar la competitividad y eficiencia de sus recursos financieros.

En la actualidad las empresas en general buscan ser más competitivas dentro de su rama adoptando estrategias que garanticen el éxito local y global basado en nuevos enfoques gerenciales y gestión de procesos, dentro de este panorama se encuentran los inventarios como parte importante de los sistemas de contabilidad, el correcto orden y administración de los mismos constituye uno de los principales factores que inciden en el desempeño de las organizaciones y en las ganancias que se obtendrán, el propósito es mantener un nivel óptimo y no generar costos innecesarios que representen un gasto mayor en el estado de resultados, Chirinos (2016). 


\section{Inventarios y su importancia en la empresa comercial}

Según Laveriano (2010), al inventario se lo puede definir como el registro fundamentado de los bienes perceptibles almacenados destinados a la venta o consumo para su comercialización. Además, se lo suele considerar un amortiguador entre dos procesos: el abastecimiento aporta bienes al inventario y la demanda los consume.

El término "inventarios" incluye los siguientes elementos Velásquez (2012): activos a ser vendidos durante el curso normal de las operaciones, activos en producción para la venta y activos sostenidos como elementos o materiales predestinados al consumo durante la fabricación de bienes o prestación de servicios, Arraiz (2018).

Los inventarios son fundamentales para mantener un control, y con estos se garantiza la posesión de información útil para minimizar costos de producción, incrementar la liquidez, y conocer confiablemente la situación financiera de la organización. Adicionalmente, se evita la insuficiencia de inventario, el robo de productos, las pérdidas, y el desorden. Sobre la base de estas ideas Arango, Giraldo, y Castrillón (2013), señalan que la importancia de los inventarios se ha incrementado, debido a su impacto en asegurar la reserva de los productos para los clientes y en las exigencias de capital que deben invertirse en las empresas. Sánchez y Señalin (2015) afirman que en toda empresa se necesita un equilibrio que garantice la menor inversión pero permitiendo el mejor nivel de servicio, cumpliendo con la satisfacción del cliente.

Sobre las bases de las ideas expuestas, para la valoración global de inventarios, los métodos son: (a) Identificación específica, (b) método primeras en entrar, primeras en salir (c) costo promedio ponderado (Velásquez, 2012).

Para la Fundación IASC (2009) el método de Identificación específica es el tipo de método que es ideal para productos que no sean periódicamente intercambiables indiferentemente si estos fueron comprados o fabricados, requiriendo de juicio profesional para la determinación de los productos destacados entre sí, además indica que este método consiste en que cada tipo de costo determinado se divide entre los productos que conforman los inventarios. 
Por otra parte, el método de valoración Primeras en Entrar, Primeras en Salir, los primeros costos que ingresan al inventario son los primeros costos que emergen al costo de las mercancías cedidas. Además, la compañía lleva un registro del costo de cada elemento adquirido del inventario. El costo de la unidad adquirida usada para el cálculo del inventario final, puede ser distinto de los costos seccionales utilizados para el cálculo del costo de los bienes vendidos (Moreno, Romero, y Membreño, 2008).

Por otro lado, el método de costo promedio ponderado, está basado en el costo promedio del inventario durante el período. Este método pondera el costo por unidad como el costo unitario promedio durante un periodo, esto es, si el costo de la unidad baja o sube durante el periodo. El costo promedio es el cociente entre el costo de los productos disponibles para la venta y el número de unidades a usar (Moreno, Romero, y Membreño, 2008).

Cabe señalar que, en los costos de inventarios, se excluyen los costos de almacenamiento posteriores a la culminación del producto, pérdidas y los costos de mercadeo. Además, se consideran todos los costos derivados de la compra y transformación, así como otros en los que haya incurrido para darles su condición y ubicación. Entonces, los costos de los inventarios según Velásquez (2012) incluyen: que los costos de adquisición tales como el precio de compra, aranceles e impuestos parecidos, transporte, almacenamiento, y otros relacionados a la adquisición de inventarios y costo de transformación: costos directos, como mano de obra y materiales; y costos indirectos, fijos y variables que hayan participado en el proceso de transformación.

Ante la importancia de los inventarios en el mundo empresarial, industrial e incluso en las empresas de servicios, y debido a la globalización de los mercados es necesario su correcto tratamiento, saber su reconocimiento, su medición, y todos los aspectos que se deben tener en cuenta. Existen diferentes clases de inventarios, entre otros como se detalla a continuación: 
Inventario Físico: Es el inventario real, permite contar, pesar o medir y anotar todas y cada una de las diferentes clases de bienes (mercancías), que se hallen en existencia en la fecha del inventario, y evaluar cada una de dichas partidas, se realiza como una lista detallada y valorada. Inventario determinado por observación y comprobado con una lista de conteo, peso. Cálculo del inventario realizado mediante un listado del stock realmente poseído. La realización de este inventario tiene como finalidad, convencer a los auditores de que los registros del inventario representan fielmente el valor del activo principal.

Inventario determinado por observación y comprobado con una lista de conteo, del peso o a la medida real obtenidos. (Juca, Narváez y Erazo, 2019)

Inventario de Previsión: Se tienen con el fin de cubrir una necesidad futura perfectamente definida. Se diferencia con el respecto a los de seguridad, en que los de previsión se tienen a la luz de una necesidad que se conoce con certeza razonable y por lo tanto, involucra un menor riesgo (Torres, Narváez, Erazo y Ormaza, 2019).

Inventarios Estaciónales: Los inventarios utilizados con este fin se diseñan para cumplir más económicamente la demanda estacional variando los niveles de producción para satisfacer fluctuaciones en la demanda. Estos inventarios se utilizan para suavizar el nivel de producción de las operaciones, para que los trabajadores no tengan que contratarse o despedirse frecuentemente.

Inventario Permanente: Método seguido en el funcionamiento de algunas cuentas, en general representativas de existencias, cuyo saldo ha de coincidir en cualquier momento con el valor de los stocks. (Balcázar, Narváez y Erazo, 2019) 
CIENCIAMATRIA

Revista Interdisciplinaria de Humanidades, Educación, Ciencia y Tecnología

Año V. Vol. V. №1. Edición Especial. 2019

Hecho el depósito de ley: pp201602FA4721

ISSN-L: 2542-3029; ISSN: 2610-802X

Universidad Nacional Experimental Francisco de Miranda (UNEFM). Santa Ana de Coro. Venezuela

Katherine Janeth Tamayo Guzmàn; Cecilia Ivonne Narvàez Zurita; Juan Carlos Erazo Alvarez

Tabla 1.

Tipos de inventarios

\begin{tabular}{|c|c|c|c|c|c|}
\hline \multicolumn{6}{|c|}{ Tipos de inventarios } \\
\hline Según el momento & $\begin{array}{l}\text { Según la } \\
\text { periodicidad }\end{array}$ & Según laforma & Según lafunción & Según la logística & Otrostipos \\
\hline mventario inicial & Inventanio irternitente & $\begin{array}{l}\text { Invertario de materias } \\
\text { primas }\end{array}$ & Inventario en társ ito & $\begin{array}{l}\text { huentariode } \\
\text { existencias para } \\
\text { espeaulación }\end{array}$ & huentariofísico \\
\hline \multirow[t]{4}{*}{ hventariofiral } & Inventario perpetuo & $\begin{array}{l}\text { Invertario de productos } \\
\text { enfabricación }\end{array}$ & Inventario de ado & $\begin{array}{l}\text { huentariode } \\
\text { existencias obs oletas }\end{array}$ & hentario míimo \\
\hline & & $\begin{array}{l}\text { Invertario de productos } \\
\text { terminados }\end{array}$ & Inventario de seguridad & mentario en ductos & mentario máximo \\
\hline & & $\begin{array}{l}\text { Invertario de } \\
\text { suministros de fábrica }\end{array}$ & Inventario de previsión & $\begin{array}{l}\text { hventario de } \\
\text { existencias de } \\
\text { seguridad }\end{array}$ & mentariodisporible \\
\hline & & $\begin{array}{l}\text { Invertario de } \\
\text { mercancias }\end{array}$ & $\begin{array}{l}\text { Inventario de } \\
\text { desacoplamierto }\end{array}$ & $\begin{array}{l}\text { huentario de } \\
\text { existencias naturaleza }\end{array}$ & mentarioen linea \\
\hline
\end{tabular}

Fuente: Emprende Pyme (2015).

\section{Método de valorización de inventarios: Identificación específica}

Para la Fundación IASC (2009) este tipo de método es ideal para productos que no sean periódicamente intercambiables indiferentemente si estos fueron comprados o fabricados, requiriendo de juicio profesional para la determinación de los productos destacados entre sí, además indica que este método consiste en que cada tipo de costo determinado se divide entre los productos que conforman los inventarios.

Por su parte, Duque, Osorio, y Agudelo (2010) consideran que el método de identificación específica es recomendable para los productos en donde los niveles de producción son bajos con costos altos y que no son habitualmente intercambiables, estos autores explican que sean productos con características propias que los hace únicos y por tanto no son intercambiables. 
Sin embargo, el método FIFO implica que las primeras mercaderías compradas o fabricadas son las primeras en venderse, valorando así el inventario final en base a los último precios; es decir si estos bajan el inventario será valorado a un precio menor y aumentará si estos se incrementan, de este modo los costos de las primeras en entrar afectan al resultado del ejercicio como costo de mercadería vendida, mientas que las últimas en entrar se reflejaran en el balance general como inventario (Duque, Osorio, y Agudelo, 2010).

Según (Escandón, Narváez, Erazo y Ormaza, 2019), una entidad calculará el costo de los inventarios, contrario a los relacionados con el procedimiento de identificación específica, utilizando el método de primera entrada, primera salida (FIFO). Asimismo, usará la fórmula para todos los inventarios que tengan uso o naturaleza similar. Para aquellos que no, pueden estar admitidas las fórmulas de costo diferentes.

La fórmula FIFO asume que los productos en almacenamiento comprados o producidos antes, serán vendidos primero y, seguidamente, que los productos que queden en el almacenamiento final serán obtenidos más recientemente.

Al utilizar el método o formula del costo promedio ponderado, el costo de cada unidad se determinará partiendo del promedio ponderado del costo de los artículos equivalentes, y del costo de estos artículos obtenidos durante la acción.

\section{Modelo de gestión de inventarios}

De acuerdo con la autora Guerrero (2005) "La palabra inglesa stock es un término utilizado en el idioma castellano, (...) stock es un término que indica un depósito de mercancías, materias primas u otro objeto cualquiera. Es un concepto estático" (p.15).

Según Pérez, Cifuentes, Vásques y Ocampo (2013) "El inventario es un recurso almacenado al que se recurre para satisfacer una necesidad actual o futura" (p.1).

Al vincular el concepto de los modelos de gestión con la definición de inventarios, se forma una herramienta útil para solucionar efectivamente el control de las existencias en una empresa y enfrentar los problemas relacionados con la falta de capacidad de 
almacenamiento, el desperdicio, el sobre stock, productos obsoletos, inconvenientes financieros, entre otros. La aplicación de un óptimo modelo de gestión del inventario incluirá métodos de análisis y control de acuerdo con la importancia económica de cada producto.

Según Mora (2017):

La gestión de los inventarios de una empresa es el último control para asegurar que no se presenten diferencias entre las existencias físicas y los registros del software, es decir, es donde se hace la validación final de que todos los procesos previos en el centro de distribución estuvieron ejecutados con base a unas buenas prácticas y de acuerdo con los procedimientos de la compañía. (p. 117)

La gestión de inventarios analizado desde un contexto estratégico de acuerdo con los autores Alonso, Di Novella, Rodriguez y Celis (2009), presenta objetivos que se encuentran en frecuente conflicto al intentar maximizar la calidad de servicio al cliente y pretender minimizar los costos por inventarios, las decisiones en torno al control de existencias son de carácter operativo y promueven estrategias para lograr la consecución de los resultados esperados.

Los modelos de gestión de inventarios son uno de los temas más complejos en la logística, puesto que siempre existe una no concordancia en lo que se vende o consume, lo cual es da por la falta de información a tiempo sobre la demanda y el consumo. La aplicación de un modelo de gestión de existencias es una alternativa para reducir costos y mejorar la economía de la empresa, puesto que incrementa el nivel de servicio al cliente, la liquidez y las fluctuaciones de la demanda, logrando mantener los inventarios necesarios para cada producto.

En cuanto a la clasificación de los modelos de inventarios, de acuerdo a Salas (2009) todo producto se clasifica en función al tipo de demanda, en este sentido puede ser de dos tipos determinística cuando se conoce con exactitud la demanda de un producto para un periodo de tiempo determinando, también puede ser probabilística cuando no se conoce con seguridad la demanda de un producto. 
De igual manera el autor establece una subclasificación dependiendo de otras condiciones relacionadas:

- Tipo de producto:

Pueden tratarse de productos con un tiempo de caducidad breve, productos sustitutos 0 de larga duración.

- Cantidad de productos:

Número de modelos de producto.

- Modelos que permiten o no deficit

Tiempos de entrega

- Modelos que involucran o no costos fijos.

- Tipo de revision:

Mantenimiento e productos

- Tipo de reposición:

Un producto puede ser de reposición instantánea cuando es comprado a un proveedor o continua cuando pasa por un proceso de producción.

- Horizonte de planeación:

Puede incluir un solo periodo o varios

\section{Beneficios de la buena gestión de inventarios}

Dentro del mundo globalizado en el que vivimos la competencia cada vez alcanza un nivel superior, las compañías no se pueden dar el lujo de perder clientes o tener el dinero amortizado en forma de inventario, el objetivo es lograr un equilibrio entre la oferta y la demanda, de igual manera alcanzar un alto grado de confiabilidad entre el tiempo de entrega de la mercadería del proveedor y a su vez el tiempo de entrega del producto al consumidor final.

Sin duda un manejo óptimo del inventario implica tomar mejores decisiones para generar mayor rentabilidad, además de otros resultados como productos en condiciones 
óptimas, clientes satisfechos, control sobre los costos, estandarización de la calidad, entre otros puntos positivos para el bienestar empresarial.

De acuerdo con la fundación de estudios profesionales FIAEP (2014), los beneficios afines con la buena gestión de inventarios están relacionados directamente con la planeación, y se analizan a continuación:

\section{Planeación de compras de la empresa}

Al conseguir un control sobre el inventario de la empresa, se crean datos y estadísticas útiles para realizar compras efectivas, descartando los excesos o faltantes, ya que todos los movimientos de los productos serán analizados, además su historial de ventas, con esto se determinará la cantidad necesaria de compra dependiendo de la época del año.

\section{Planeación del flujo de efectivo}

Con el manejo eficiente de los inventarios se conseguirá realizar un pronóstico del flujo del efectivo óptimo, lo más cercano a la realidad, de igual manera se conocerá cuánto invertir, montos de compra, y fechas estimadas, que permitirán eliminar ciertos gastos imprevistos, no propios a la operación que ponen en riesgo la liquidez de la organización.

Los autores consideran que la gestión vinculada al control de los inventarios constituye un proceso de gran impacto en el área operativa administrativa y financiera de la empresa, puesto que conservar un producto en bodega por mucho tiempo implica un costo muy alto, además de una inversión mayor de activo circulante, la empresa debe apuntar a establecer mecanismos que controlen esta situación y la regulen para bienestar de la compañía. 


\section{METODOLOGÍA}

El enfoque bajo el cual se desarrolló esta investigación fue mixto, por cuanto en su ejecución se obtuvo información cuali - cuantitativa en un solo momento de tiempo (finalidad transversal), la cual sirvió de insumo para comprender de mejor manera la problemática de la unidad analizada. Con un alcance descriptivo - explicativo, se fundamentó teóricamente la normativa referente a inventarios y se detalló la aplicación del método de valorización más recomendable para la empresa.

Su diseño fue experimental, dado que se analizaron los datos tal cual fue la realidad de la empresa. Mediante la aplicación del método histórico - lógico se cimentó la revisión bibliográfica de acuerdo a la relevancia de la información respecto de las pautas establecidas en las normas sobre los inventarios; además se empleó el método analítico - sintético al descomponer el objeto de estudio y posterior análisis de sus componentes de forma integral, se utilizó también el método inductivo - deductivo vinculando la teoría de los modelos de gestión de inventarios.

La unidad de análisis de la presente investigación fue la empresa "Agroinsumos SV" localizada en la ciudad de Machala provincia de El Oro, Ecuador; donde se realizó la aplicación de instrumentos de investigación a su personal (entrevista y encuestas); y a sus 18 diferentes productos que conforman el inventario (observación y revisión documental); sin la aplicación de tratamiento de la muestra.

\section{RESULTADOS}

Los principales resultados obtenidos en la fase de diagnóstico de la investigación evidencian que:

Toma física de inventarios: el $75 \%$ de los encuestados manifiestan que respecto a la determinación de existencias no hay periodicidad en la toma física de inventarios, que ha existido algunos años en los que se realizó una vez y en otros ninguna.

Manuales de procedimientos: el $100 \%$ manifiesta que no existen procedimientos escritos que direccionen el manejo de los inventarios y que permitan desarrollar una 
gestión eficiente, razón por la cual mencionan que existen tantos problemas con las existencias de la empresa objeto de estudio de esta investigación.

Auditoría a los procesos de inventarios: el 100\% menciona que no hay procesos de auditoria que permitan identificar pérdidas de insumos por robo, caducidad etc. Esto genera además de pérdidas económicas, insatisfacción de los clientes al no encontrar en stock los productos que necesitan.

Evaluación de entradas y salidas de mercadería: el 100\% coincide en que el manejo es totalmente empírico y sin documentación de respaldo como registros de entrada y salida de la mercadería que permitan al menos establecer responsabilidades del mal manejo de inventarios.

Conteos cíclicos: el 100\% establece la necesidad de que existan conteos cíclicos que permitan reabastecer los productos y que se pueda determinar responsabilidades respecto al mal manejo.

De acuerdo a lo expuesto por el Gerente se requiere implementar procesos de capacitación y entrenamiento en el manejo de inventarios y el establecimiento de técnicas cuantitativas como definición de niveles máximos y mínimos de existencias.

Se realizó también un análisis documental in situ valorando del 1 al 5, siendo 1 el mas bajo.

En función a los resultados obtenidos en la investigación y con el objeto de plantear un manejo efectivo de los inventarios se debe considerar dos funciones básicas relativas al control y manejo de las existencias, por medio de la aplicación de métodos, estrategias o herramientas que aporten a generar más rentabilidad, productividad y desarrollo empresarial, las funciones mencionadas son las siguientes:

- Determinación de las existencias: Involucra juntar toda la informacion referente a las existencias y ejercer control sobre los procesos operativos inherentes a esta actividad, desde la toma de inventarios hasta los conteos frecuentes requeridos. 


\section{CIENCIAMATRIA}

Revista Interdisciplinaria de Humanidades, Educación, Ciencia y Tecnología

Año V. Vol. V. №1. Edición Especial. 2019

Hecho el depósito de ley: pp201602FA4721

ISSN-L: 2542-3029; ISSN: 2610-802X

Universidad Nacional Experimental Francisco de Miranda (UNEFM). Santa Ana de Coro. Venezuela

Katherine Janeth Tamayo Guzmàn; Cecilia Ivonne Narvàez Zurita; Juan Carlos Erazo Alvarez

- Análisis de los inventarios: Realizar analisis estadisticos, enfocado a la rentabilidad que pueden producir dichas existencias, aplicando metodologias como la "formula de wilson" y el "just in time" para lograr este fin.

Figura 1.

Propuesta de modelo de gestion de los inventarios para la Empresa Agroinsumos SV

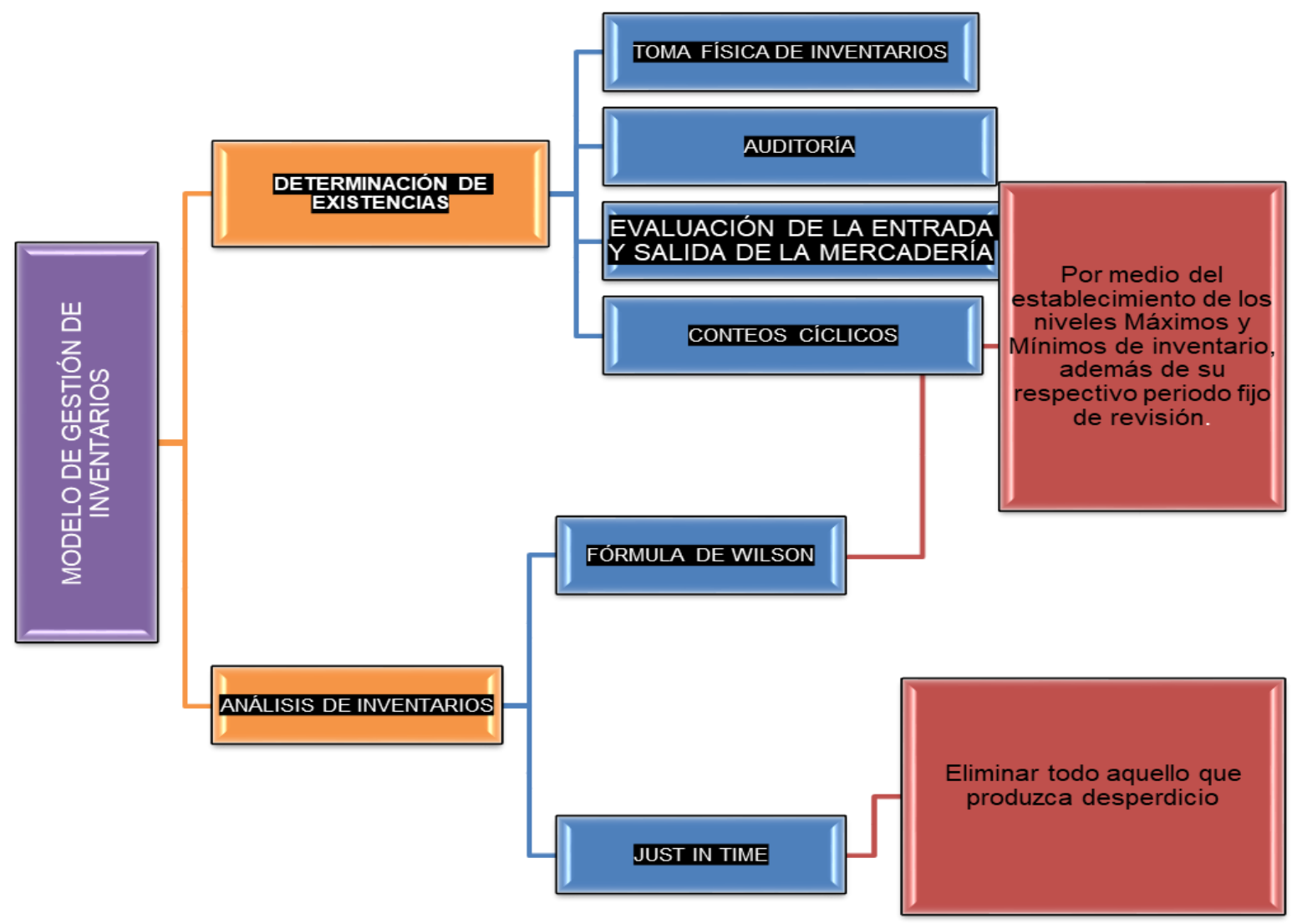




\section{Existencia Máxima}

Es una cantidad máxima que económicamente puede mantenerse en stock de cada producto, para evitar posibles desabastecimientos, se determina a través del consumo máximo y el tiempo de reposición, en este caso utilizamos como ejemplo el consumo máximo mensual del producto con código L09-03 y el tiempo máximo de remplazo en el mercado es de 25 días.

$\begin{aligned} \text { Existencia Máxima }= & \frac{\text { Consumo tiempo máximo }}{30 \text { dias }} \times \text { días reemplazo } \\ \text { Existencia Máxima }= & \frac{120}{30 \text { días }} \times 25\end{aligned}$

Existencia Máxima $=\quad 100$ unidades

\section{Existencias Mínimas}

Es el nivel mínimo de stock que debe permanecer en existencias para que los procesos de ventas no se interrumpan, se calcula considerando los consumos periódicos y el tiempo de reposición, en este caso el consumo mínimo mensual es de 50 unidades del producto con código L09-03 y el tiempo mínimo de reposición en el mercado es de 16 días.

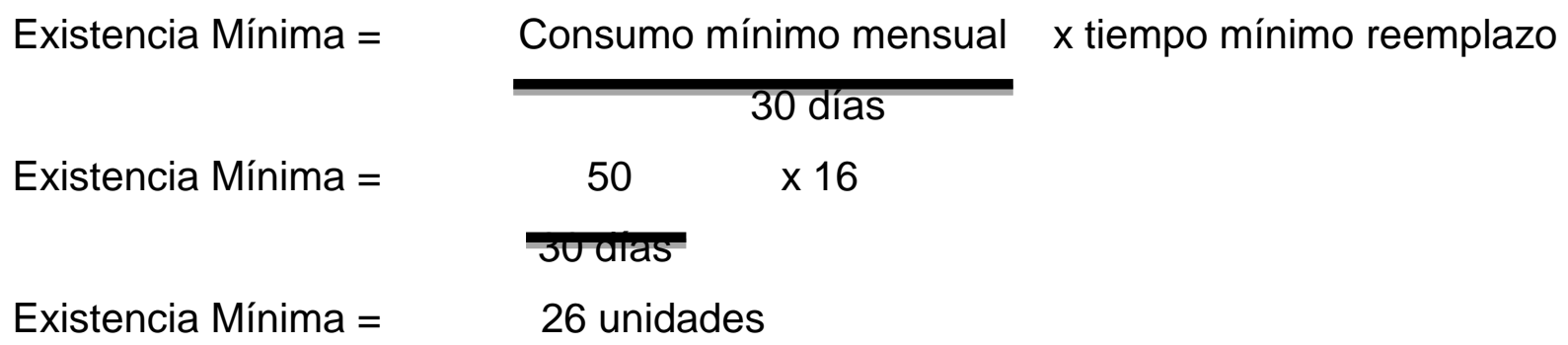

\section{Existencias Críticas}

Es el nivel más bajo al que puede llegar el stock, en el caso de que fallen los abastecimientos normales de los proveedores, una vez llegando a ese nivel es necesario activar los mecanismos extraordinarios de adquisición de mercaderías para 
que la empresa no se paralice en su actividad, en este caso el consumo mínimo mensual es de 50 unidades y el tiempo crítico de reposición es de 3 días.

Existencia Crítica $=$

Existencia Crítica $=$
Consumo mínimo mensual x tiempo mínimo reemplazo

$$
30 \text { días }
$$

$\frac{50}{30 \text { días }} \times 3$

Existencia Crítica $=\quad 5$ unidades

\section{CONCLUSIONES}

El control de inventarios permitirá minimizar el riesgo de la inversión, manteniendo stocks adecuados y suficientes, así como minimizar los costos de almacenamiento.

La importancia del inventario consiste en suministrar a la empresa la mercadería necesaria para la comercialización en el momento adecuado y a un bajo costo, esto permitirá también mejorar la satisfacción de los clientes.

La toma física de los inventarios realizado en forma periódica permite a la empresa mantener datos reales de las existencias en bodega, con esto se puede comprobar con los datos del software contable, determinando en qué momento abastecer de mercadería.

Un adecuado modelo de gestión de inventarios como el que se plantea además de los beneficios antes mencionados permitirá mejorar la competitividad y eficiencia de sus recursos financieros. 


\section{REFERENCIAS CONSULTADAS}

1. Alonso, A., Di Novella, P., Rodriguez, M., \& Celis, P. (2009). UN ESTUDIO DE LA GESTIÓN DE INVENTARIOS EN VENEZUELA. Revista de la Facultad de Ingeniería U.C.V, 84.

2. Arraiz, A. (2018). Framerwork de desarrollo de Proyectos Sociotecnológicos basado en la notación de Metamodelos de procesos de Ingeniería de Software (spem 2.0). Revista Arbitrada Interdisciplinaria Koinonía, 3(6), 79-95. Recuperado de

https://fundacionkoinonia.com.ve/ojs/index.php/revistakoinonia/article/view/141/1 $\underline{21}$

3. Balcázar Sarmiento, B. M., Narváez Zurita, C. I., \& Erazo Álvarez, J. C. (2019). Herramientas de contabilidad gerencial para la toma de decisiones financieras en la empresa Tapitex M\&B Cía. Ltda. de la ciudad de Cuenca. Visionario Digital.

4. Chirinos, A. (2016). La innovación como factor clave de éxito en la gerencia de las empresas de producción social venezolana. CIENCIAMATRIA, 2(2), 97-106. https://doi.org/10.35381/cm.v2i2.55

5. Editoriales Expreso EC. (06 de 10 de 2014). Obtenido de El productor: http://elproductor.com/noticias/las-empresas-de-ecuador-prefieren-el-comercio-yla-agricultura/

6. Escandón Guillén, P. d., Narváez Zurita, C. I., Erazo Álvarez, J. C., \& Ormaza Andrade, J. E. (2019). La gestión de costos basada en actividades como herramienta estratégica para la toma de decisiones en las empresas constructoras de la ciudad de Loja, Ecuador. Visionario Digital, 375.

7. FIAEP, F. I. (2014). Control y manejo de inventario y Almacén. Fundación Iberoamericana de altos estudios profesionales FIAEP.

8. Guerrero Parra, F. (2005). Gestión de stocks. Madrid: Esic Editorial.

9. Juca Tello, C. E., Narváez Zurita, C. I., \& Erazo Álvarez, J. C. (2019). Modelo de gestión y control de inventarios para la determinación de los niveles óptimos en la cadena de suministros de la Empresa Modesto Casajoana Cía. Ltda. 593 Digital Publisher CEIT, 21.

10. Laveriano, W. (2010). Importancia del control de inventarios en la empresa. Actualidad 
http://biblioteca.iplacex.cl/RCA/Importancia\%20del\%20control\%20de\%20inventar ios\%20en\%20la\%20empresa.pdf

11. Mora García, L. A. (2017). Gestión de Logística en centros de distribución, bodegas y almacenes. Bogotá: Ecoe ediciones.

12.Pérez Vergara, I., Cifuentes Laguna, A. M., Vásques García, C., \& Ocampo, D. M. (2013). Un modelo de gestión de inventarios para una empresa de productos alimenticios. Ingeniería Industrial/ISSN, 228.

13.Pyme, E. (2015). https://www.emprendepyme.net/tipos-de-inventarios.html. Obtenido de https://www.emprendepyme.net/tipos-de-inventarios.html

14. Salas Guerrero, H. (2009). Inventarios, Manejo y Control. Bogotá: Ecoe ediciones.

15. Superintendencia de Compañías. (2008). RESOLUCION No. 08.G.DSC. Quito.

16. Superintendencia de Compañías. (2008). RESOLUCION No. 08.G.DSC. Guayaquil.

17. Superintendencia de Compañías. (2011). Resolución № SC.ICI.CPAIFRS.G.11.010. Guayaquil.

18. Torres Flores, M. E., Narváez Zurita, C. I., Erazo Álvarez, J. C., \& Ormaza Andrade, J. E. (2019). Costos ABC/ABM como herramienta de gestión empresarial. Caso empresa PlanERP Cía. Ltda. Visionario Digital, 121.

19. Velásquez, R. (2012). Implementación de las NIIF'S - NIC 1 presentación de estados financieros, NIIF'S 1 adopción de las NIIF'S por primera vez, NIC 2 inventarios, NIC 16 activos fijos, para empresas comerciales dedicadas a la venta de repuestos de tractocamiones. Quito: Universidad Politécnica Salesiana. Obtenido de https://dspace.ups.edu.ec/handle/123456789/4079

\section{REFERENCES CONSULTED}

1. Alonso, A., Di Novella, P., Rodriguez, M., \& Celis, P. (2009). A STUDY OF INVENTORY MANAGEMENT IN VENEZUELA. Magazine of the Faculty of Engineering U.C.V, 84. 
2. Arraiz, A. (2018). Framerwork of development of Sociotechnological Projects based on the notation of Metamodels of Software Engineering processes (spem 2.0). Interdisciplinary Arbitrated Review Koinonía, 3 (6), 79-95. Recovered from https://fundacionkoinonia.com.ve/ojs/index.php/revistakoinonia/article/view/141/1 $\underline{21}$

3. Balcázar Sarmiento, B. M., Narváez Zurita, C. I., \& Erazo Álvarez, J. C. (2019). Management accounting tools for financial decision making at Tapitex M\&B Cía. Ltda. Of the city of Cuenca. Digital Visionary

4. Chirinos, A. (2016). Innovation as a key success factor in the management of Venezuelan social production companies. SCIENCE, 2 (2), 97-106. https://doi.org/10.35381/cm.v2i2.55

5. Editorial Express EC. (06 of 10 of 2014). Obtained from The producer: http://elproductor.com/noticias/las-empresas-de-ecuador-prefieren-el-comercíyla-agricultura/

6. Escandón Guillén, P. d., Narváez Zurita, C. I., Erazo Álvarez, J. C., \& Ormaza Andrade, J. E. (2019). Activity-based cost management as a strategic tool for decision-making in construction companies in the city of Loja, Ecuador. Digital Visionary, 375.

7. FIAEP, F. I. (2014). Control and management of inventory and warehouse. Iberoamerican Foundation of high professional studies FIAEP.

8. Guerrero Parra, F. (2005). Inventory management. Madrid: Esic Editorial.

9. Juca Tello, C. E., Narváez Zurita, C. I., \& Erazo Álvarez, J. C. (2019). Inventory management and control model for the determination of optimal levels in the supply chain of Modesto Casajoana Cía. Ltda. 593 Digital Publisher CEIT, 21.

10. Laveriano, W. (2010). Importance of inventory control in the company. Business News. Retrieved from http://biblioteca.iplacex.cl/RCA/Importancia\%20del\%20control\%20de\%20inverabl es\%20en\%20la\%20empresa.pdf

11. Mora García, L.A. (2017). Logistics management in distribution centers, warehouses and warehouses. Bogotá: Ecoe editions. 
12.Pérez Vergara, I., Cifuentes Laguna, A. M., Vásques García, C., \& Ocampo, D. M. (2013). An inventory management model for a food products company. Industrial Engineering / ISSN, 228.

13. Pyme, E. (2015). https://www.emprendepyme.net/tipos-de-inventario.html. Obtained from https://www.emprendepyme.net/tipos-de-inventario.html

14. Salas Guerrero, H. (2009). Inventories, Management and Control. Bogotá: Ecoe editions.

15. Superintendence of Companies. (2008). RESOLUTION No. 08.G.DSC. Quito

16. Superintendence of Companies. (2008). RESOLUTION No. 08.G.DSC. Guayaquil

17. Superintendence of Companies. (2011). Resolution No. SC.ICI.CPAIFRS.G.11.010. Guayaquil

18. Torres Flores, M. E., Narváez Zurita, C. I., Erazo Álvarez, J. C., \& Ormaza Andrade, J. E. (2019). ABC / ABM costs as a business management tool. Case of PlanERP Cía. Ltda. Digital Visionary, 121.

19. Velásquez, R. (2012). Implementation of IFRS '- IAS 1 presentation of financial statements, IFRS' 1 adoption of IFRS 'for the first time, IAS 2 inventories, IAS 16 fixed assets, for commercial companies dedicated to the sale of truck parts. Quito: Salesian Polytechnic University. Retrieved from https://dspace.ups.edu.ec/handle/123456789/4079 